\title{
Non-Contact Luminescence Lifetime Microthermometry using Scintillation Sensors
}

\author{
V.B. MykhaylyK ${ }^{a, *}$, H. KRAus ${ }^{b}$ And A. WAgner ${ }^{a}$ \\ ${ }^{a}$ Diamond Light Source Ltd, Harwell Science Campus, Didcot, OX11 0DE, UK \\ ${ }^{b}$ Department of Physics, University of Oxford, Keble Road, Oxford OX1 3RH, UK
}

\begin{abstract}
The paper describes an original technique for non-contact in situ monitoring of the temperature developed for experiments in a vacuum environment at the tender X-ray beamline I23 of the Diamond Light Source. The sample temperature is established by determining the luminescence decay constant of a $\mathrm{Bi}_{4} \mathrm{Ge}_{3} \mathrm{O}_{12}$ (BGO) scintillation sensor. BGO is ideally suited for a temperature range of $30 \mathrm{~K}$ to $130 \mathrm{~K}$, in which its decay constant shows a strong temperature dependence. The principle of the method, system design and application examples are discussed and the performance and potential of the technique is assessed.
\end{abstract}

DOI: 10.12693/APhysPolA.133.1108

PACS/topics: 78.70.Ps

\section{Introduction}

Temperature is an important parameter when describing certain properties of systems in nature. Knowledge of accurate temperature is required when monitoring chemical, physical, and biological processes. For example, temperature is of high importance in developing a strategy for reducing radiation damage to protein crystals during macromolecular crystallography (MX) experiments, using intense synchrotron radiation [1]. It is currently well established that the ability of biological samples to withstand radiation damage increases by two orders of magnitude when cooled below the temperature of the phase transition from vitreous to crystalline ice, typically occurring above $130 \mathrm{~K}[2]$. Therefore, cooling the protein samples to cryogenic temperatures $(c a .100 \mathrm{~K})$ by a nitrogen cold-gas stream is currently standard practice in MX experiments. This method of cooling ensures that the temperature of a protein crystal placed inside the stream remains equal or close to the temperature of the cooling gas for the duration of the experiment [3].

Recent progress in synchrotron instrumentation has created a new opportunity for MX experiments. Extending experimental capabilities towards lower X-ray energy offers a direct method for solving the crystallographic phase problem by utilizing the anomalous scattering signals observed close to the absorption edges of light atoms, naturally occurring in proteins and nucleic acids. Due to adverse absorption and scattering of low-energy X-rays the experiment ought to be conducted in vacuum which brings about a number of major technical challenges. The success of an X-ray diffraction experiment in a vacuum environment relies critically on the efficient cooling of protein samples. The common technique adopted for cooling of experimental samples in vacuo employs a series

*corresponding author; e-mail: vmikhai@hotmail.com of highly conductive sections that provide a good thermal link to a cooler $[4,5]$. Many factors affect the thermal conductivity and subsequently the temperature of the experimental sample. These include the properties of materials used (thermal conductivity), geometry (distance and cross-section) as well as presence of additional components (interfaces and interposers). Therefore, monitoring the temperature of cooled samples of macromolecular crystals in a vacuum environment is an important task that requires innovative technical solutions.

A novel technique for remote, non-contact temperature monitoring has been developed for the new I23 beamline at the Diamond Light Source, a unique facility dedicated to MX experiments with low energy X-rays [6]. The beamline operates in a vacuum and accurate knowledge of the sample temperature is very important as there is an increased possibility of protein sample heating by intense X-ray radiation. Conceptually the thermometry method is based on measurements of the variation of the luminescence lifetime of a scintillation crystal with temperature. In this paper we introduce the basics of the developed technique, present results on system characterisation and discuss the first data of in situ temperature measurements obtained using the non-contact thermometry system.

\section{Principles of luminescence cryothermometry}

Luminescence in dielectric materials originates from the radiative recombination of electrons promoted to excited levels with holes in the valence band. The transition to the ground state can occur via either radiative or non-radiative processes. In the simplest two-level system, composed of a ground and an excited state, the luminescence lifetime $\tau$ is defined as the inverse of the sum of two rates:

$$
\tau=\left(w_{r}+w_{n r}\right)^{-1}
$$

where $w_{r}$ and $w_{n r}$ are rates for radiative and nonradiative processes. The rate associated with the non- 
radiative processes $\left(w_{n r}\right)$ exhibits a strong temperature dependence, thus controlling a variation of the decay time constant with temperature. The simplest case is the thermal quenching of the luminescence when electrons can be promoted over the energy gap $\Delta E$ and then recombine non-radiatively. This results in a decrease of the measured luminescence decay time constant with temperature $T$ expressed by

$$
\tau^{-1}=\tau_{r}^{-1}+K \exp (-\Delta E / k T)
$$

where $\tau_{r}=w_{r}^{-1}$ is the radiative decay constant, $K$ is the non-radiative decay rate and $k$ is the Boltzmann constant. The temperature dependence of the decay time constant given by this equation is modelled in Fig. 1 (curve a). The figure illustrates that for a two-level system the temperature-dependent relaxation channel dominates at high temperatures, determining the range in which the decay time constant can be useful for temperature monitoring.

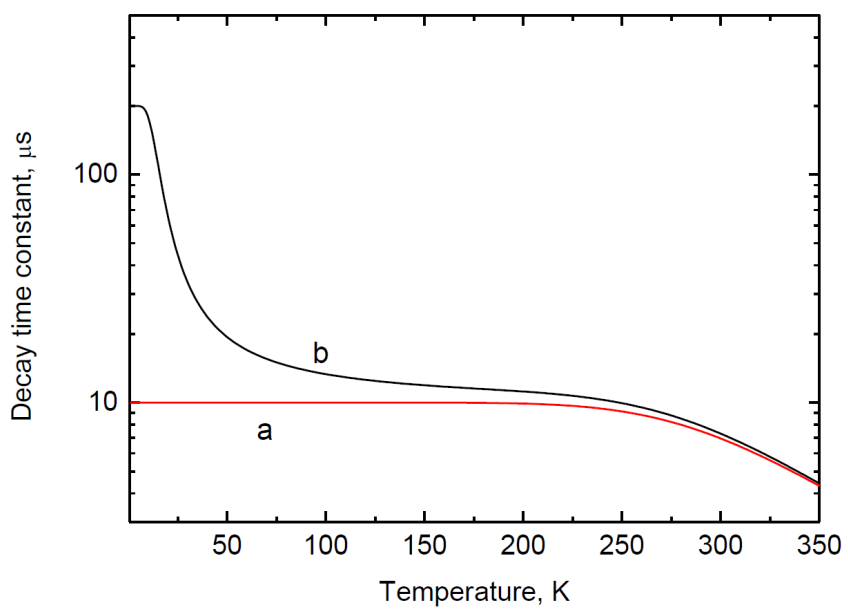

Fig. 1. Luminescence decay time constants as function of temperature, calculated using the two models discussed in the text. The parameters for the two-level model (a, Eq. (2)) are: $\tau_{r}=10^{-5} \mathrm{~s}, K=10^{8} \mathrm{~s}^{-1}$ and $\Delta E=0.2 \mathrm{eV}$. The parameters for the three-level model (b, Eq. (3)) are: $w_{1}=5 \times 10^{3} \mathrm{~s}^{-1}, w_{2}=2 \times 10^{5} \mathrm{~s}^{-1}$, $D=0.005 \mathrm{eV} K=10^{8} \mathrm{~s}^{-1}$ and $\Delta E=0.2 \mathrm{eV}$. The used parameters are adapted from [8].

In some materials, the structure of energy levels of the emission centres facilitates a complementary mechanism resulting in variation of the decay time constant at lower temperatures. Thus in a three-level system composed of a ground state and two excited levels, separated by a narrow energy gap $D$, the temperature dependence of the decay time constant is given by [7]:

$$
\tau^{-1}=\frac{w_{1}+w_{2} \exp (-D / k T)}{1+\exp (-D / k T)}+K \exp (-\Delta E / k T) .
$$

Here, $w_{1}$ and $w_{2}$ are the rates of radiative decays from the two excited levels. Figure 1 (curve b) shows the temperature dependence of the decay time constant calculated according to Eq. (3). At low temperatures the relaxation process is governed by the dynamics of transitions between two excited levels, leading to a change of the measured decay time constant. As is demonstrated by curve $b$ in Fig. 1 this effect causes significant variation of the luminescence decay time constant over a wide temperature range. This feature is key for the high sensitivity of the measurement technique at cryogenic temperatures down to a few kelvins. It has been shown recently that this feature is fairly common in self-activated scintillation oxides [8]. For our application, we have chosen $\mathrm{Bi}_{4} \mathrm{Ge}_{3} \mathrm{O}_{12}$ (BGO) as scintillator. This material exhibits good sensitivity over a broad range of temperatures [9].

\section{Measurement system}

The thermometry system was designed as integral part of the I23 experimental end station with the main hardware components integrated in the viewing system (see Fig. 2). Mirror M3 is used to switch from sample visualisation to thermometry by directing photons towards the photodetector. An ultraviolet LED driven by a waveform generator produces rectangular pulses of light with $\lambda=245 \mathrm{~nm}(\Delta \lambda=12 \mathrm{~nm}$, frequency of $200 \mathrm{~Hz})$ used for luminescence excitation. A splinter of a BGO scintillator (sensor) is attached to the sample holder, loaded into a vacuum vessel with the pressure is $\left\langle 10^{-7} \mathrm{mbar}\right.$, placed in the receptacle of the cryogenic goniometer and positioned in the field of view of the viewing system. Luminescence produced by the scintillator is detected by a photomultiplier tube (PMT). The signal from the PMT is processed and analysed using the multiphoton counting technique [10]. The temperature is then derived from the value of the decay time constant using the calibration curve displayed in Fig. 3. In this case, the uncertainty in determining temperature was assessed to be $\pm 1.6 \mathrm{~K}$.

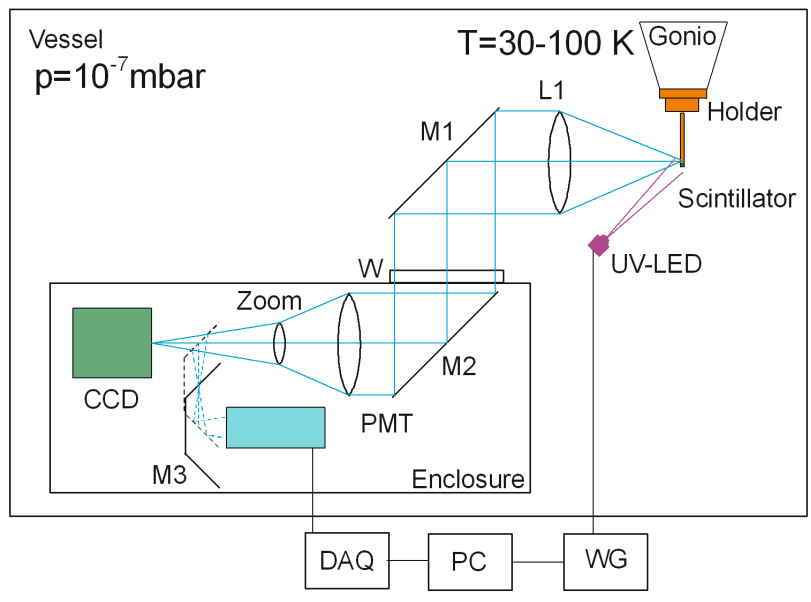

Fig. 2. Schematics of the luminescence lifetime thermometry system: M1-M3 - mirrors, L1 - objective lens, UV LED - ultraviolet light emitting diode, W - optical window, PMT - photomultiplier tube, CCD - camera, DAQ - data acquisition, PC - personal computer, WG - waveform generator. 


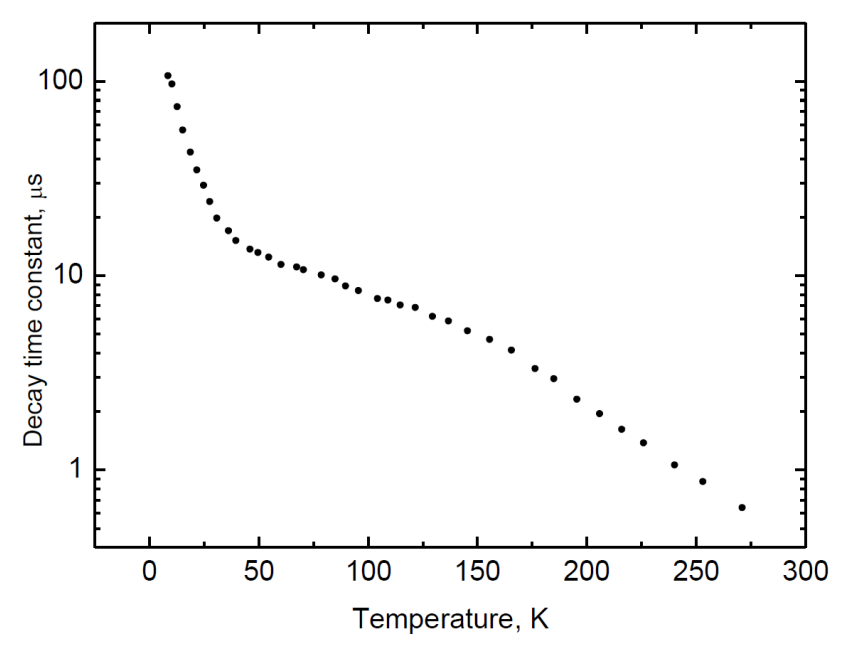

Fig. 3. Luminescence decay time constant as function of temperature for the BGO scintillator at UV excitation $(\lambda=245 \mathrm{~nm})$.

\section{System testing}

To verify the suitability of the thermometry system, we measured in a first test the temperature of a sample holder mounted in the receptacle of the cryogenic goniometer. In stationary conditions the sample holder, made of highly thermally conductive copper, remains at the same temperature that can be derived from the known temperature of the goniometer. A splinter of BGO sensor was affixed to the end of the sample holder pin by means of Apiezon $\mathrm{N}$ grease and luminescence decay curves were recorded using the thermometry system. The temperature of the BGO crystal measured in this way was found to be equal to $41.1 \pm 0.9 \mathrm{~K}$. The temperature of the goniometer receptacle, measured using a platinum thermometer PT-100, was 38.6 K. According to [11] the expected temperature increment across the interface between the sample holder and the receptacle is $3 \mathrm{~K}$. Hence, both estimated $(38.6 \mathrm{~K}+3 \mathrm{~K}=41.6 \mathrm{~K})$ and measured $(41.1 \pm 0.9 \mathrm{~K})$ temperatures agree well within the error margin. This test demonstrated that the developed luminescence lifetime thermometry system provides fairly accurate measurements of temperature.

Of principal interest and importance is the determination of temperature of the dielectric sample mount that is used to support the protein sample during XRD experiments. It is made of thin kapton with low X-ray absorption and shaped as a circular loop on a stem (see Fig. 4). Due to the nature of the material used it has low thermal conductivity e.g. $0.1-0.2 \mathrm{~W} /(\mathrm{m} \mathrm{K})$ at $50 \mathrm{~K}$. Therefore, we used the non-contact thermometry for characterisation of thermal performance of the sample holder with the mount made of $25 \mu \mathrm{m}$ thick kapton film. For this test to be representative, it is essential to reproduce the environment of the experiments with the protein crystals when the samples are flash-frozen in a cryoprotecting liquid. Grains of a pulverised BGO crystal ( were immersed in a drop of $20 \%$ glycerol-water solution, a typical cryoprotectant (antifreeze) used in MX. A sample mount was dipped in the drop to fish the grains coated by solution from the drop together with excess of liquid (see Fig. 4, top). Samples were then flash-frozen in liquid nitrogen and transferred to the goniometer receptacle that was kept at a temperature of $47.5 \pm 0.5 \mathrm{~K}$. The goniometer translations allow different parts of the sample mount to be placed in the field of view while the zoom module defines a small region of interest permitting detection of luminescence from the selected region. When the loop is illuminated by UV light from the LED, the bright bluegreen emission typical for BGO can be clearly seen using the beamline viewing system (see Fig. 4, bottom).
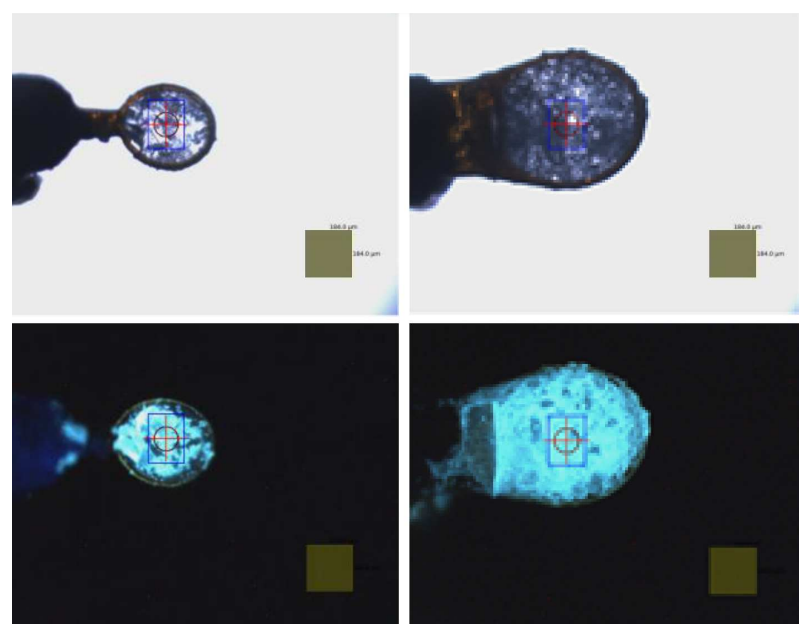

Fig. 4. Photograph of the BGO grains distributed over the loops of the sample mounts (top). The same under UV illumination (bottom). The loop diameter is $300 \mu \mathrm{m}$ (left) and $500 \mu \mathrm{m}$ (right). The squares in the corner of the pictures have dimensions $184 \mu \mathrm{m} \times 184 \mu \mathrm{m}$.

The variation of temperature along the loop is displayed in Fig. 5. The results show that cooling efficiency is better for the $500 \mu \mathrm{m}$ loop as its absolute temperature is lower in comparison to that of the $300 \mu \mathrm{m}$ loop. It is also noticeable that the temperature gradient across the loop ( $c a .5 \mathrm{~K}$ ) remains fairly similar in both cases. Qualitatively these findings can be explained as follows. The loop is linked to the copper pin through the stem section. As is shown in Fig. 4, for the $300 \mu \mathrm{m}$ loop the stem is much narrower, by about factor of four. Consequently, the heat transport through this part of the assembly is significantly impeded. That explains the higher temperature detected for the $300 \mu \mathrm{m}$ loop. When loops are filled by the vitrified cryoprotectant it homogeneously fills the gaps between the BGO grains and provides a medium for heat transport with higher thermal conductivity $(0.6 \mathrm{~W} /(\mathrm{m} \mathrm{K}))$. Therefore, the temperature exhibits a fairly smooth variation across the loop. Modelling of the heat flow through the sample holder assembly is now in preparation that should provide a quantitative interpretation of these experimental 
results. This experiment demonstrates the potential of the non-contact thermometry system for investigations of the temperature distribution with sub-millimetre spatial resolution.

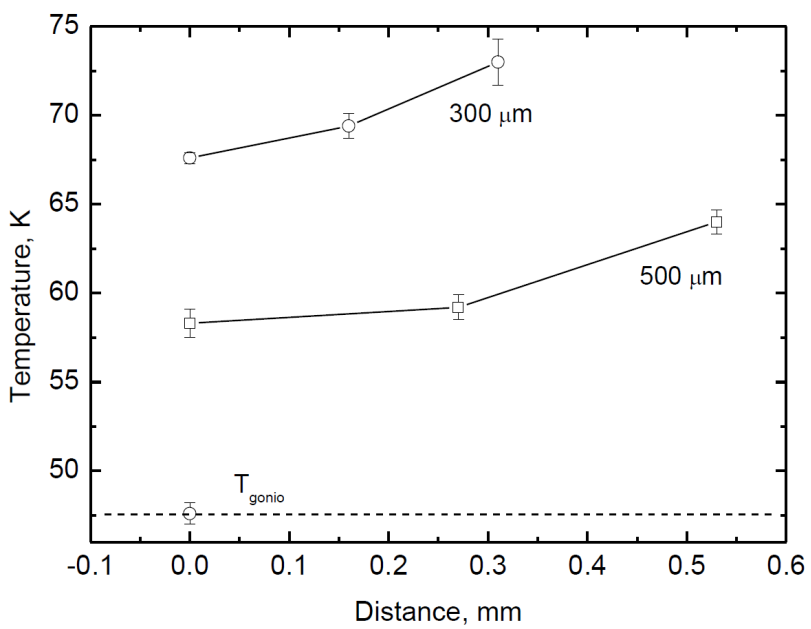

Fig. 5. Variation of temperature across the 300 and $500 \mu \mathrm{m}$ loops of the sample mount, made of a $25 \mu \mathrm{m}$ thick kapton film. Zero represents the point where the loop merges with the stem. $T_{\text {gonio }}$ is the temperature of the goniometer receptacle during measurements.

\section{Conclusions}

Based on a technique developed earlier for studies of cryogenic scintillators we designed a method for remote, non-contact measurements of temperatures in a cryogenic vacuum environment. The temperature is derived from the changes in the luminescence decay constant of a scintillation sensor. The main advantage of the non-contact thermometry system is the elimination of any connection between the sensor and the readout system, which makes it fully compatible with the vacuum environment of the beamline, and necessity of swift replacement and manipulation (transfer, mounting, rotation) of the samples.

In this work, we described the basic features of the cryogenic luminescence lifetime thermometry starting from an introduction of the measurement principle that exploits the peculiarities of radiative decay observed in self-activated scintillators with the three-level energy structure of emission centres. We discussed the main components and features of practical implementation of the system into a suite of beamline instrumentation. The technique was tested using a bismuth germanate scintillator sensor, exhibiting a pronounced change of decay time constant at cryogenic temperatures. The BGO sensor was calibrated over the $8-270 \mathrm{~K}$ temperature range and the accuracy of the measurements was evaluated. It is found that in the temperature range of beamline operation $(30-150 \mathrm{~K})$ the error of temperature determination is $\pm 1.6 \mathrm{~K}$. The technique was applied to characterise the temperature rise across the sample holder assembly used in the MX experiment at the I23 beamline. The information gained in such studies will aid optimisation of the sample holder assembly.

\section{Acknowledgments}

This work was supported by the Science and Technology Facilities Council (STFC) UK through grant ST/K002929.

\section{References}

[1] E.F. Garman, M. Weik, J. Synchr. Radiat. 24, 1 (2017).

[2] M. Weik, J.-P. Colletier, Acta Crystallogr. D 66, 437 (2010).

[3] E.H. Snell, H.D. Bellamy, G. Rosenbaum, M.J. van der Woerd, J. Synchr. Radiat. 14, 109 (2007).

[4] A. Al-Amoudi, L.P.O. Norlen, J. Dubochet, J. Struct. Biol. 148, 131 (2004).

[5] A. Kobayashi, Y. Sekiguchi, T. Oroguchi, K. Okajima, A. Fukuda, M. Oide, M. Yamamoto, M. Nakasako, J. Synchr. Radiat. 23, 975 (2016).

[6] A. Wagner, R. Duman, K. Henderson, V. Mykhaylyk, Acta Crystallogr. D 72, 430 (2016).

[7] X. Zhang, J. Lin, V.B. Mikhailik, H. Kraus, Appl. Phys. Lett. 106, 241904 (2015).

[8] V.B. Mikhailik, H. Kraus, J. Phys. Stud. 14, 4201 (2010).

[9] J. Gironnet, V.B. Mikhailik, H. Kraus, P. de Marcillac, N. Coron, Nucl. Instrum. Methods Phys. Res. A, 594, 358 (2008).

[10] V.B. Mikhailik, H. Kraus, Radiat. Measur. 49, 7 (2013).

[11] V. Mykhaylyk, A. Wagner, J. Phys. Conf. Ser. 425, 012010 (2013) 